\title{
Latent developmental potential to form limb-like skeletal structures in zebrafish
}

M. Brent Hawkins ${ }^{1,2,3,4}$, Katrin Henke ${ }^{1,2}$, and Matthew P. Harris ${ }^{1,2}$

${ }^{1}$ Department of Genetics, Harvard Medical School, Boston, MA, USA

${ }^{2}$ Department of Orthopedic Research, Boston Children's Hospital, Boston, MA, USA

${ }^{3}$ Department of Organismic \& Evolutionary Biology, Harvard University, Cambridge, MA, USA

${ }^{4}$ Museum of Comparative Zoology, Harvard University, Cambridge, MA, USA

Corresponding Author:

Matthew P. Harris

harris@genetics.med.harvard.edu

Department of Genetics, Harvard Medical School

Orthopedic Research Laboratories

Boston Children's Hospital

300 Longwood Ave Enders 260

Boston, MA 02115 


\section{Abstract}

The evolution of fins into limbs was a key transition in vertebrate history. A hallmark of this transition is the addition of multiple long bones to the proximal-distal axis of paired appendages. Whereas limb skeletons are often elaborate and diverse, teleost pectoral fins retain a simple endoskeleton. Fins and limbs share many core developmental processes, but how these programs were reshaped to produce limbs from fins during evolution remains enigmatic. Here we identify zebrafish mutants that form supernumerary long bones along the proximal-distal axis of pectoral fins with limb-like patterning. These new skeletal elements are integrated into the fin, as they are connected to the musculature, form joints, and articulate with neighboring bones. This phenotype is caused by activating mutations in previously unrecognized regulators of appendage development, vav2 and waslb, which we show function in a common pathway. We find that this pathway functions in appendage development across vertebrates, and loss of Wasl in developing limbs results in patterning defects identical to those seen in Hoxal1 knockout mice. Concordantly, formation of supernumerary fin long bones requires the function of hoxal1 paralogs, indicating developmental homology with the forearm and the existence of a latent functional Hox code patterning the fin endoskeleton. Our findings reveal an inherent limb-like patterning ability in fins that can be activated by simple genetic perturbation, resulting in the elaboration of the endoskeleton.

\section{Introduction}

Changes in appendage structure underlie key transitions in vertebrate evolution. Tetrapod limbs permit mobility via articulation of multiple endochondral long bones facilitated by specialized synovial joints. Diverse limb structures and functions have evolved in tetrapods through digit reduction, phalangeal addition, transformation of wrist and ankle bones, alteration in element proportion, and outright limb-loss. In contrast to limbs, the skeletal pattern of the teleost pectoral appendage is almost invariant, showing a consistent arrangement across diverse lineages within this group of $\sim 30,000$ species. Teleost pectoral fins are composed of dermal fin rays supported at their base by a diminutive endoskeleton (Fig. 1A). The endoskeleton typically consists of four long bones, called proximal radials, arranged side by side along the anteriorposterior (AP) axis, followed distally by small nodular distal radials $(1,2)$. No known teleost 
1 species exhibits more than a single long bone along the proximal-distal (PD) axis (3). The

2 divergent architectures of teleost fins and tetrapod limbs have been maintained within each clade

3 for over $250(4,5)$ and 350 million (6) years, respectively.

4 Evidence from fossils suggests that, similar to extant ray-finned fishes, the common

5 ancestor of teleosts and tetrapods had a series of long bones arranged side by side along the AP

6 axis of the pectoral appendage (7). The posterior-most element of this series was segmented

7 along the PD axis, creating end-on-end articulation (Fig. 1A). This structure, called the

8 metapterygium, is not retained in teleost fishes, and is hypothesized to have been elaborated in

9 lobe-finned fishes to form limbs (8) (Figure S1). The progressive acquisition and regionalization

10 of elements along the PD axis of the appendicular skeleton in stem and crown group lobe-finned

11 fishes indicate that the limb is a unique feature in this group not present in ray-finned fishes.

Due to their divergent skeletal patterns, identifying homologous structures between fins

13 and limbs with one-to-one correspondence is difficult. However, despite the morphological

14 disparity between fins and limbs, comparative approaches in developmental genetics have

15 revealed shared pathways required for the normal patterning of these appendages. Hox genes, in

16 particular, are key players that specify and reinforce regional identity in appendages (9).

17 Overexpression of hoxd13a in embryonic zebrafish causes increased, but unpatterned,

18 chondrogenic tissue formation in the larval pectoral fin (10). Furthermore, deletions of posterior

19 group 13 HoxA and HoxD genes result in the loss of distal skeletal elements in mouse limbs

20 (wrist/ankle and hand/foot) and zebrafish fins (dermal fin rays), suggesting that these genes

21 provide a regional address for distal elements in the different appendages (11). While Hox genes

22 that specify more proximal limb components have been identified in tetrapods, it is unclear if

23 similar mechanisms instruct the patterning of non-distal components of the fin.

24 Here, through unbiased forward genetic screens in the zebrafish $(12,13)$, we identify

25 mutants that break the long-standing teleost fin pattern by developing new skeletal elements

26 along the PD axis of the pectoral fin in a limb-like manner. These novel structures are fully

27 differentiated long bones that integrate into the fin musculature and articulate with the fin

28 skeleton. Mapping and epistasis analysis reveal modulation of vav2 and waslb signaling as

29 causing the mutant phenotypes, and that these genes function together in a common pathway not

30 previously associated with limb development or embryonic patterning. Conditional knockout of

31 Wasl in the mouse demonstrates that this pathway is required for normal limb patterning, and its 
1 absence results in a range of skeletal phenotypes that match those seen in Hoxal1 knockout

2 mutants. Through generation of triple loss-of-function hox11 zebrafish mutants by CRISPR-Cas9

3 gene editing, we demonstrate that waslb requires hox 11 function to form supernumerary

4 elements, and more broadly interacts with hox genes to pattern the PD fin axis. Our results

5 suggest that zebrafish retain the capacity to form structures with an intermediate regional address

6 specified by hoxa11, similar to the limb zeugopod. This inherent capacity is activated by simple

7 genetic changes, revealing a novel appendage-patterning pathway that may have played

8 unrecognized roles in the fin to limb transition.

\section{Results}

\section{Breaking the teleost fin ground plan}

Wild-type zebrafish pectoral fins are representative of the ancestral teleost configuration,

14 with four proximal radials arranged side-by-side followed by small nodular distal radials (Fig.

15 1B). All four proximal radials are in direct contact with the shoulder early in development, but

16 the two posterior radials shift distally in adulthood (14). To identify genes affecting the form and

17 pattern of the adult skeleton in zebrafish, we conducted a forward mutagenesis screen focused on

18 dominant mutations (13). In our screen, we isolated a mutant that breaks from the teleost ground

19 plan by forming supernumerary long bones along the PD axis of the pectoral fin endoskeleton

20 (Fig. 1C). We call these new bones 'intermediate radials.' They are found between the posterior

21 proximal radials and distal radials, and they do not articulate directly with the shoulder. The

22 overall pattern and size of the fin rays are not dramatically altered. We named this mutant

23 rephaim (reph). The mutant phenotype exhibits varied expressivity and dose sensitivity, as

24 homozygous fish are highly dysmorphic (Figure S2). Due to the severity of the homozygous

25 condition, we have focused our analysis on heterozygous reph mutants.

\section{Uncovering novel fin patterning genes}

28 Using whole-exome sequencing, we mapped the reph mutation to chromosome 4 (Figure

$29 \mathrm{~S} 3$ ) (15). Analysis of sequence data from the linked interval identified a missense mutation in the

30 gene wiskott-aldrich syndrome-like b (waslb), changing a serine to a proline at amino acid

31 position 265 (Fig. 1D). Waslb is a member of the WAS protein family of actin cytoskeleton

32 signaling regulators, which interact with $\mathrm{Cdc} 42$ and Arp2/3 to nucleate filamentous actin 
1 formation, and have roles in cell migration, cell signaling, and cell polarity (16). Additionally,

2 nuclear-localized Wasl is required for the transcription of select genes through its interaction

3 with nuclear actin, transcription factors, and RNA polymerase $(17,18)$.

4 To verify that the waslb mutation causes the reph phenotype, as well as to determine the

5 genetic nature of the dominant reph allele, we used CRISPR-Cas9 to generate targeted frameshift

6 mutations in wild-type waslb as well as in cis to the candidate reph S265P mutation, and

7 assessed their effect on fin skeleton patterning (Fig. 1D, Figures S4, S5). Loss-of-function of

8 wild-type waslb does not have any obvious effect on fin patterning. However, frameshift

9 mutations generated upstream in cis to the missense mutation lead to reversion of the mutant

10 phenotype, restoring the wild-type radial pattern. Small, in-frame deletions in cis to the mutation

11 failed to rescue the wild-type phenotype. Together, these results demonstrate that the identified

12 S265P mutation in waslb causes the reph phenotype, likely through a gain-of-function effect.

13 The altered residue in reph is conserved across vertebrates and is positioned in a hinge region

14 that is thought to regulate auto-inhibition and localization of the protein (19). Consistent with

15 this, introduction of the reph mutation into fluorescently tagged Wasl protein led to a marked

16 reduction in nuclear localization of the fusion protein in cell culture (Figure S5).

\section{Genetic interactors of Wasl signaling}

As waslb is a novel factor in appendage development, we sought to identify additional loci that affect fin endoskeleton patterning in a similar manner. Although large dominant screens are not common in zebrafish, mutants having viable dominant phenotypes have been identified as a by-product of recessive genetic screens. We searched published screens and identified a

23 mutant called wanda (wan) with a phenotype similar to reph (Figure S2) (12, 20). We obtained

24 the wan mutant and found that it exhibited intermediate radials in a pattern similar to reph (Fig.

25 1E), a phenotype that had not been previously reported. Similar to reph, wan exhibits variable

26 phenotypic expressivity and dose sensitivity (Figure S2).

We mapped wan and identified a missense mutation in a conserved residue of the vav2

28 gene on chromosome 21 (S67A; Fig. 1D, Figure S3). The Vav family of protooncogenes

29 function as guanosine nucleotide exchange factors for Rho GTPases, and have roles in signal

30 transduction, cytoskeletal regulation, cell motility, and receptor endocytosis (21-23). We created

31 vav2 loss-of-function mutations using CRISPR-Cas9 and found that vav2 homozygous null 
1 zebrafish are viable and have no skeletal phenotypes (Figures S4, S5), consistent with the

2 phenotype reported for the $\operatorname{Vav} 2$ knockout mouse (24). In addition, truncating mutations made in

3 cis upstream of the S67A wan mutation cause reversion of the wan skeletal phenotype (Fig. 1D,

4 Figure S5). Thus, similar to reph, wan causes fin patterning changes through a gain-of-function

5 mutation.

Vav2 activates the small G-protein Cdc42 (25), and Cdc42 in turn regulates the activity of Wasl (26). As such, it is likely that the similar phenotypes observed in reph and wan mutants

8 are due to their effect on a common signaling pathway. To test this hypothesis, we crossed waslb

9 loss-of-function alleles to the wan mutant and found that waslb function is required for the

10 expression of the wan phenotype (Fig. 1E). Thus, waslb is epistatic to vav2, and these genes act

11 together in a common pathway to pattern the fin endoskeleton (Fig. 1F). Whole mount in situ

12 hybridization reveals that both genes are expressed in fins during early patterning stages (Fig.

$131 \mathrm{G})$, suggesting that these genes might act in the developing fin mesenchyme. Interestingly,

14 neither waslb nor vav2 have previously been associated with fin or limb development,

15 skeletogenesis, or embryonic patterning at large.

\section{Fin fold integrity is unchanged in rephaim}

18 In the fin to limb transition, there was a reduction of the dermal skeletal fin rays concomitant with the elaboration of the endoskeleton, until fin rays were ultimately lost in crown tetrapods. Fin rays arise from the larval fin fold (27). Mechanistic hypotheses regarding the finto-limb transition posit a developmental trade-off between the fin endoskeleton and the fin fold,

22 such that early formation of the fin fold in teleosts results in their diminutive endoskeleton (28).

23 Previous studies have tested if reduction of the fin fold can result in limb-like characteristics in

24 developing zebrafish fins. Experimental perturbation of the fin fold during early development

25 leads to an enlargement of the endoskeletal disc in larval zebrafish, suggesting that the fin fold

26 represses disc growth $(29,30)$. However, similar to the effect of reducing hox 13 paralog function

27 (11), subsequent elaboration of the adult fin endoskeleton is generally negligible, or has not been

28 assessed, in the different manipulations. To determine if changes in fin fold dynamics underlie

29 intermediate radial development, we examined fin fold formation in $\mathrm{reph}^{+/-}$mutants (Fig. 2).

30 Immunolabeling of actinodin, the collagens that support the larval fin fold, revealed no

31 differences between wild-type and reph $^{+/-}$pectoral fins at 3 days post fertilization (dpf; Fig. 2A, 
1 B). We also find no difference in endoskeletal disc size at $7 \mathrm{dpf}$ (Fig. 2C-E). The absence of

2 changes in the endoskeletal disc and fin fold is consistent with a lack of patterning defects in 3-

3 week-old mutant fins (Fig. 2F, G). Thus, contrary to previous hypotheses, changes to fin fold

4 development are not required for PD elaboration of the endoskeleton in reph $^{+/-}$mutants.

\section{Formation of intermediate radials in rephaim}

In teleost fishes, the fin endoskeleton first forms as a continuous endoskeletal disc of cartilage that is subsequently subdivided into the four proximal radial anlagen by localized involution and trans-differentiation, followed by perichondral ossification (Fig. 2F, H) $(14,31)$.

10 This process occurs normally in reph ${ }^{+/-}$mutants (Fig. 2G). It is only after initial patterning and

11 differentiation that differences are seen between wild-type and mutant fish endoskeletons, when

$12 \mathrm{reph}^{+/-}$mutants show an elongation of the more posterior cartilage condensations compared to

13 wild-type fish (Fig. 2I). The extended condensations in $\mathrm{reph}^{+/-}$mutants form multiple sites of

14 mineralization, with the nascent intermediate radial forming a distinct bone collar (Fig. 2K),

15 while wild-type siblings have a continuous zone of mineralization beginning proximally and

16 growing distally (Fig. 2J). The elongated condensation in mutants is subsequently segmented,

17 resolving into two distinct bones separated by joint territories. This mode of ossification and

18 cartilage segmentation in $r e p h^{+/-}$mutants has not been reported in the pectoral fins of any other

19 teleost, and is similar to tetrapod limb development, where an initially continuous condensation

20 is segmented along the PD axis to produce independent elements separated by a joint (32).

\section{Morphological integration of an expanded fin skeleton}

Histological analysis of adult fins reveals that, while proximal radials possess epiphyses

24 only at their distal ends, reph intermediate radials form both proximal and distal epiphyses (Fig.

$253 \mathrm{~A}, \mathrm{~B})$. Both distal and proximal epiphyses exhibit normal differentiation of the cartilage in

$26 \mathrm{reph}^{+/-}$mutants. Like the epiphyses of wild-type proximal radials and the long bones of tetrapod

27 limbs, the newly formed epiphyses of intermediate radials express bone patterning genes such as

28 indian hedgehog a (ihha; Fig. 3C) (33). Intermediate radials also show evidence of vascular

29 invasion (Fig. 3D), suggesting the activation of endochondral ossification programs in reph ${ }^{+/}$

30 mutants similar to those found in tetrapod long bones and the bones of larger fishes (34). In

31 limbs, synovial joints are formed between long bone elements (32). Similarly, in reph ${ }^{+/-}$mutants, 
1 proximal and intermediate radials form a distinct joint pocket between them with specialized

2 differentiation of mesenchyme shaping the interface (Fig. 3E). We find that the terminal joint

3 marker $\operatorname{prg} 4 b(35,36)$ is expressed in the superficial chondrocytes of epiphyses, as well as in

4 cells lining the joint space (Fig. 3F). These findings suggest that the joint differentiation

5 programs found in limbs are also activated in the reph intermediate radials.

In limbs, muscles originate from the shoulder as well as the limb bones and insert on

7 more distal positions along the appendage (Fig. 3G). Zebrafish, in contrast, have seven muscles

8 that originate on elements of the shoulder girdle and insert directly on the dermal fin rays,

9 bypassing the fin long bones entirely (37). This configuration of the pectoral fin musculature is

10 representative of most teleost fishes, and only in certain derived lineages does a muscle insert on

11 pectoral fin radial bones (38). Surprisingly, reph $^{+/-}$intermediate radials exhibit novel insertion

12 points from muscles originating from the shoulder (Fig. 3B). Thus, not only does the reph

13 mutation reveal a capacity to make differentiated and patterned long bones, but these elements

14 are morphologically integrated to form limb-like joints and muscle connections not seen in the

15 fins of other teleosts.

\section{Wasl is required for normal limb patterning and axial identity in tetrapods}

Both wasl and vav2 have not previously been implicated in the regulation of developmental patterning. To assess if the patterning roles of vav2/waslb signaling are zebrafish

20 specific, or if they are conserved across bony fishes, we asked if Wasl is required for normal

21 tetrapod limb development. As homozygous somatic knockout of Wasl in mouse is embryonic

22 lethal (39), preventing analysis of adult limb patterning, we generated mice with conditional loss

23 of Wasl function in developing limb progenitors by crossing the Prrx 1-Cre driver (40) into a

24 floxed Wasl $^{\mathrm{L} 2 \mathrm{~L}}$ background (41). Wasl ${ }^{\mathrm{L} 2 \mathrm{~L} /+}$ and Wasl $^{\mathrm{L} 2 \mathrm{~L} / \mathrm{L} 2 \mathrm{~L}}$ animals lacking the Cre driver are

25 phenotypically wild type, as are $\operatorname{Wasl}^{\mathrm{L} 2 \mathrm{~L} /+}$ heterozygotes with the driver (Fig. 4A-D,

26 Supplemental Table 1). However, Wasl $^{\text {L2L/L2L }}$; Prrxl-Cre limb knockout (LKO) mice exhibit

27 dramatic defects in limb and axial patterning. The long bones of LKO mouse limbs are shorter

28 and wider than those of wild-type siblings (Fig. 4A, C). LKO mice also exhibit various coalitions

29 of the carpal bones (Fig. 4B), while the tibia and fibula fail to fuse (Fig. 4C). Axial homeosis

30 occurs in LKO mice, where the first sacral vertebra is transformed to exhibit partial or complete

31 lumbar identity (Fig. 4D). This demonstrates that Wasl is necessary for proper patterning in 
1 limbs and the vertebral column. Intriguingly, this syndrome of axial and appendicular

2 phenotypes closely matches those resulting from somatic loss of Hoxal1 function (42), a gene

3 thought to have played a key role in the fin to limb transition.

5 Waslb regulates appendage patterning through hoxa function

It is unclear if intermediate radials have a specific positional identity defined by hox gene expression, as is observed in the elements of tetrapod limbs. Limbs have a tripartite bauplan that has been canalized through their evolutionary history (Fig. 1), consisting of one long bone (humerus) in the upper arm (the stylopod), two side-by-side long bones (radius and ulna) in the forearm (the zeugopod), and many nodular and long bones in the wrist and hand (the autopod).

11 Differential expression of Hox genes along the growing limb is essential for specification of 12 positional identity and growth (9). The A and D members of Hox paralogy group 13 are

13 expressed in the autopod, while the A and D members of group 11 are expressed in the zeugopod

14 (43-45). Loss of either group results in the reduced growth or even absence of the elements in

15 which they are expressed $(46,47)$. The pectoral fins of teleosts and other ray-finned fishes do not

16 exhibit comparable hox regulation, as hox 11 and hox 13 paralogs are expressed in overlapping

17 domains (48-50). This has been thought to be consistent with the simple adult skeleton formed in

18 teleost fins (48). Interestingly, hox13 genes are however required for the development of the

19 distal fin rays (11).

We asked if intermediate radials formed through activation of waslb signaling bear a

21 positional identity specified by a Hox code similar to that found in limbs. Based on the Wasl

22 LKO mouse phenotype, we hypothesized that reph might interact with hoxall paralogs. To test

23 this, we created null alleles of the hoxalla, hoxal1b, and hoxd11a genes and examined how loss

24 of these factors affects the expression of the reph phenotype. Homozygous null mutants for all

25 three paralogs are viable and fertile, singularly and in combination, and do not show obvious

26 phenotypes in the formation of the fin skeleton (Fig. 4G, Figure S6). However, in reph ${ }^{+/-}$

27 mutants, loss of hoxal1 paralog function resulted in the loss of intermediate radials, reverting the

$28 \mathrm{reph}^{+/-}$pectoral skeleton to the wild-type pattern (Fig. 4H). This result suggests that the

29 intermediate radials formed in $\mathrm{reph}^{+/-}$mutants share a zeugopodial-type identity, and

30 developmental homology, with the radius and ulna. Our findings demonstrate that, in contrast to

31 the roles of hox13 paralogs in regulating formation of distal fin ray structures (Fig. 4I) (11), 
1 hox 11 paralogs do not have essential roles in normal patterning of the zebrafish fin. Rather, the

2 functional necessity of hoxal1 paralogs is revealed only in the context of intermediate radial

3 formation in $\mathrm{reph}^{+/-}$mutants.

4 The Hox 13 group genes can repress the activity of more proximal Hox genes in a process

5 termed posterior prevalence (51). In limbs, Hoxal3 suppresses Hoxal1 activity, and these genes

6 form mutually exclusive expression domains at the stylopod/autopod boundary (52). Consistent

7 with the broad retention of genomic architecture of the Hox complex across vertebrate evolution

8 (53) and the requirement of hox 11 function to form reph-mediated supernumerary elements, we

9 found that loss of hox 13 function resulted in the development of additional supernumerary long

10 bones, but only in the context of waslb gain-of-function caused by the reph mutation (Fig. 4J).

11 The necessity of hoxall paralogs for the expression of the reph $^{+/}$phenotype reveals the

12 presence of a functional Hox code and regulatory network (53) within developing teleost

13 pectoral fins capable of specifying intermediate domains similar to that found in limbs. This

14 suggests that the intermediate radials have a hox11 identity and share developmental homology

15 with the zeugopodial structures of the tetrapod limb (Fig. 4K). However, the manifestation of

16 this positional code is not normally expressed in teleost fishes, but is revealed by the action of

17 the reph and wan mutations.

\section{Discussion}

The integration and differentiation of the novel long bones formed in reph and wan

22 pectoral fins is unique amongst teleost fishes, and harkens to the development and anatomy of

23 long bones in the limbs of tetrapods. The common ancestor of all bony fishes, including humans,

24 had a pectoral fin skeleton that consisted of multiple long bones arranged side by side, each

25 articulating with the shoulder (Figure S1) $(7,8)$. This polybasal condition of the endoskeleton

26 consisted of unsegmented elements in the middle and anterior portions of the appendage, and the

27 posterior metapterygium, which branched and was segmented along the PD axis. It is

28 hypothesized that the tetrapod limb skeleton evolved via loss of the anterior components and

29 elaboration of the metapterygium. Teleosts, on the other hand, are thought to have maintained

30 the polybasal condition and lost the metapterygium (54-56). Interestingly, the skeletal

31 elaboration seen in reph and wan is restricted to the posterior region of the pectoral skeleton,

32 resembling the morphology of basal actinopterygian and lobe-finned fishes $(1,57)$. Thus, it is 
1 possible that teleosts retain a cryptic ancestral axis of growth, corresponding to the metapterygial 2 axis, whose potential for elaboration is revealed by these mutants.

3 It is unlikely that the mutations identified in this study are specifically instructive of limb-

4 ness, but are instead permissive of endogenous limb-like developmental programs latent in

5 fishes. A component of these mutation-activated programs is a functional Hox code, retained in

6 teleost fishes, with specific intermediate (hoxa11) and distal (hoxa13) patterning cues. Our

7 results reveal latent or emergent properties of development within vertebrate appendages to form

8 elaborate, articulated skeletal structures. These patterning processes were present in the bony fish

9 ancestor and potentially refined in the evolution of lobe-finned fishes during the transition to

10 land. 
1 Methods

3 Zebrafish husbandry, strains, and mutagenesis

4 All zebrafish lines were maintained and propagated as described by Nuesslein-Volhard \& Dahm

5 (58). This study was conducted with ethical approval from the Institutional Animal Care and Use

6 Committee of Boston Children's Hospital. A complete description of the husbandry and

7 environmental conditions for the fish used in these experiments is available as a collection in

8 protocols.io $\mathrm{dx}$.doi.org/10.17504/protocols.io.mrjc54n. rephaim (dmh22) was isolated as part of

9 a broad mutagenesis screen performed at Boston Children's Hospital (13). wanda (ty127) was

10 initially isolated and described in the original Tuebingen screens (12). wanda mutant stocks were

11 obtained from the European Zebrafish Resource Center (http://www.ezrc.kit.edu/).

13 Mapping rephaim and wanda mutant zebrafish

14 Whole-exome sequencing was used to map the loci and mutations causing the reph and wan

15 phenotypes through homozygosity-by-descent methods (15). As both mutants exhibit dose-

16 dependent phenotypes, homozygotes could be isolated and used for mapping. For each mutant,

17 genomic DNA from $25 \mathrm{~F}_{2}$ homozygous mutants stemming from a WIK outcross was extracted

18 from whole larvae using the Qiagen DNeasy Blood \& Tissue kit. $3 \mu \mathrm{g}$ of pooled DNA was

19 sheared to an average fragment size of $200 \mathrm{bp}$ using a Covaris E220 Focused Ultrasonicator.

20 Barcoded sequencing libraries were prepared from fragmented DNA using the KAPA Hyper

21 Prep Kit following the manufacturer's protocol. We used Agilent SureSelect DNA target

22 enrichment baits designed using the Zv9 genome assembly to enrich for coding regions of the

23 zebrafish genome (59). 50 bp single-end sequencing was performed on an Illumina HiSeq

24 machine, resulting in average exome coverage of $7 \mathrm{x}$ for rephaim and 13x for wanda. Fine

25 mapping of linked intervals was performed with polymerase chain reaction (PCR)-based analysis

26 of recombinants using unique single nucleotide polymorphisms (SNP) and simple sequence

27 length polymorphisms (SSLP). Mapping PCR primers are listed in Supplemental Table 2.

29 Gene editing and isolation of frameshift mutations

30 CRISPR-Cas9 site-directed mutagenesis was used to generate null alleles for each target gene.

31 Suitable target sites located early in the first exon of each gene were selected using CHOPCHOP

32 (http://chopchop.cbu.uib.no/). 
1 hoxa11a_1: 5'ACTGGCACATTGTTATCCGT 3'; hoxa11b_1: 5' CGTCTTCTTGCCCCATGACA 3';

2 hoxa11b_2: 5' TTTGATGAGCGGGTACCCGT 3'; hoxd11a_1: 5' CGCTTCGTACTATTCAACGG 3';

3 hoxd11a_2: 5' CTATTCTTCGAaCATAGCGC 3'; vav2_1: 5' CTGAGGGCCAAACGACCCGG 3';

4 vav2_2: 5' TGGAGGAGTGGAGGCAGTGC 3'; waslb_1: 5' ATAGAGCCAACATTGAGCGC 3';

5 waslb_2: 5' AGAGCACTTCGTTTTCCTGA 3'; waslb_3: 5' GCGGTCCACCTTCAAACAGG 3'.

7 Guides were synthesized by IDT (Integrated DNA Technologies, Coralville, IA) for use with

8 their Alt-R CRISPR-Cas9 system. Gene-specific guides were multiplexed and injected at a

9 concentration of $6.25 \mu \mathrm{M}$ each crRNA guide and $500 \mathrm{ng} / \mu \mathrm{L}$ Cas9 mRNA into single-cell

10 zebrafish embryos. Progeny of injected fish were screened for the presence of inherited lesions

11 resulting in frameshifts and truncations, and these progeny were used as founders of stable

12 mutant lines. Genotyping primers and strategies for each allele are reported in Supplemental

13 Table 2. Amino acid sequence alterations caused by each lesion are reported in Supplemental

14 Table 3.

$16 \quad$ Skeletal staining and histology

17 Adult fish were fixed overnight at room temperature with agitation in $3.7 \%$ formaldehyde in

18 phosphate buffered saline (PBS) $\mathrm{pH}$ 7.4. After fixation, fish were rinsed briefly with PBS and

19 stepped through one-hour ethanol/distilled water washes, with $30 \%, 50 \%, 70 \%, 95 \%$, and finally

$20100 \%$ ethanol. Cartilage was then stained overnight at room temperature with $0.015 \%$ Alcian

21 Blue GX in 30\% acetic acid in ethanol. One-hour ethanol/distilled water washes were then used

22 to move the animals through $100 \%, 70 \%, 50 \%, 30 \%$ ethanol, and then washed twice in tap

23 water. Fish were then macerated using $0.15 \%$ trypsin in $65 \%$ saturated sodium borax solution at

$2437^{\circ} \mathrm{C}$. Typically fish were digested for 1 to 3 hours, stopping the process once the caudal

25 peduncle and anal fin radials were visible when held up to light. Mineralized tissues were stained

26 with $0.25 \%$ Alizarin Red $\mathrm{S}$ in $0.5 \% \mathrm{KOH}$ (potassium hydroxide) overnight. If one night of

27 staining was inadequate, a second overnight wash was performed with fresh staining solution.

28 For storage and imaging, stained fish were then moved through a glycerol $/ 0.5 \% \mathrm{KOH}$ series

29 using overnight washes of $20 \%, 40 \%, 60 \%, 80 \%$ glycerol in $0.5 \% \mathrm{KOH}$, and finally $100 \%$

30 glycerol. Larval and juvenile fish were stained with the same protocol, with the exception that 
1 larval fish were left in the cartilage stain for only 4 hours. All wash volumes are $20 \mathrm{~mL}$ and all

2 steps were performed in scintillation vials.

4 Calculations of endoskeletal disc area

5 Wild-type and $\mathrm{reph}^{+/} 7 \mathrm{dpf}$ larvae were stained with Alcian blue. After staining, pectoral fins

6 were removed and the endoskeletal disc was measured using integrated measurement tools in the

7 Nikon Imaging Software. For each fin, the disc area was measured in triplicate and the average

8 taken to be used in statistical analysis. Statistical comparison of average disc size was performed

9 by Welch's Two Sample $t$-test in the R statistical module (60).

11 Whole-mount actinotrichia collagen labeling

12 Whole-mount immunolabeling of actinodin collagen filaments and DAPI counter-labeling was

13 carried out as previously described by Lalonde and Akimenko $(2018)(29,30)$.

\section{Histology}

16 Fish were fixed overnight in 3.7\% formaldehyde in PBS at room temperature. After fixation, the

17 pectoral girdle was removed and decalcified in 10\% EDTA (ethylenediaminetetraacetic acid) for

18 several days, with daily solution changes. Following decalcification, fins were embedded in

19 paraffin using standard practices. Hematoxylin and Eosin staining was performed using standard

20 protocols.

22 In situ hybridization

23 Whole mount in situ hybridization was performed following Jackman et al. (61) with minor

24 modifications. Embryos were pre-treated with $2.5 \mu \mathrm{g} / \mathrm{mL}$ proteinase $\mathrm{K}$ for 30 minutes at room

25 temperature. In situ hybridization on sections was performed following Smith et al. (62). Prior to

26 embedding in OCT (Optimal Cutting Temperature, Sakura), adult fins were decalcified overnight

27 in $10 \%$ EDTA pH 7.6 .

29 Cell localization constructs and assay

30 The gene fusion construct mEmerald-N-Wasp-C-18, consisting of an N-terminal GFP fused to

31 the Mus musculus Wasl coding region driven by the CMV promoter, was a gift from Michael 
1 Davidson (Addgene plasmid \#54199). Site-directed mutagenesis via the QuikChange II XL kit

2 (Agilent) was used to introduce the S249P mutation with the primers

3 5'CCCAGCTTAAAGACAGAGAAACCTCAAAAGTTATTTATGACTTTATTG'3 and

4 5'CAATAAAGTCATAAATAACTTTTGGTGTTTCTCTGTCTTTAAGCTGGG'3. HeLa cells

5 were seeded on 6-well plates in Dulbecco's Modified Eagle's medium (DMEM, Life

6 Technologies) supplemented with 10\% fetal bovine serum (FBS, Life Technologies) and $1000 \mathrm{U}$

7 penicillin/streptomycin (Life Technologies). Cells were transfected using FuGENE transfection

8 reagent (Promega) with either $1 \mu \mathrm{g}$ wild-type or S249P construct. Two days after transfection,

9 cells were given fresh media, stained with NucBlue ${ }^{\text {TM }}$ Live Cell Stain (Molecular Probes), and

10 photographed using a Zeiss EVOS imaging system.

$12 \quad$ Mouse husbandry and breeding

13 Mice were maintained in the animal resources facilities at Boston Children's Hospital. This work

14 was conducted with the ethical approval of the Institutional Animal Care and Use Committee at

15 Boston Children's Hospital. Adult mice homozygous for the conditional Wasl allele (Wasl ${ }^{\mathrm{L} 2 \mathrm{~L}}$ )

16 were a gift from Dr. Scott Snapper. These mice were crossed to the Prrx 1-Cre driver line. All

17 resulting progeny were phenotypically wild type, and those carrying the driver transgene were

18 both in-crossed to one another and crossed to Wasl $^{\mathrm{L} 2 \mathrm{~L} / \mathrm{L} 2 \mathrm{~L}}$ homozygotes. Offspring genotypes

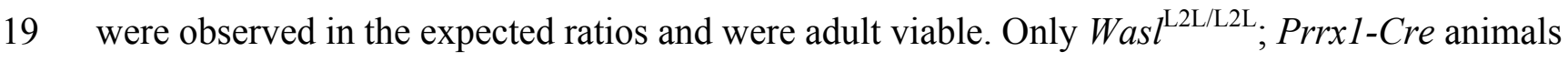

20 expressed an abnormal phenotype. 


\section{References}

1. H. L. Jessen, in Fossils and Strata, A. Martinsson, Ed. (Oslo, 1972), pp. 1-101.

2. G. Arratia, in Mesozoic Fishes 2 - Systematics and Fossil Record, G. Arratia, H. P. Schultze, Eds. (Verlag, Muchen, Germany, 1999), pp. 265-334.

3. E. C. Starks, The Primary Shoulder Girdle of the Bony Fishes. Biological Science (Stanford University Press, Stanford 1930), vol. 6.

4. R. R. Betancur et al., Phylogenetic classification of bony fishes. BMC Evol Biol 17, 162 (2017).

5. T. J. Near et al., Resolution of ray-finned fish phylogeny and timing of diversification. Proc Natl Acad Sci U S A 109, 13698-13703 (2012).

6. J. D. Pardo, M. Szostakiwskyj, P. E. Ahlberg, J. S. Anderson, Hidden morphological diversity among early tetrapods. Nature 546, 642-645 (2017).

7. M. Zhu, X. Yu, Stem sarcopterygians have primitive polybasal fin articulation. Biol Lett, 372-375 (2009).

8. C. Gegenbaur, Untersuchungen zur vergleichenden Anatomie der Wirbeltiere. (Wilheim Engelmann, Leipzig, 1865), vol. 2.

9. J. Zakany, D. Duboule, The role of Hox genes during vertebrate limb development. Curr Opin Genet Dev 17, 359-366 (2007).

10. R. Freitas, C. Gomez-Marin, J. M. Wilson, F. Casares, J. L. Gomez-Skarmeta, Hoxd13 contribution to the evolution of vertebrate appendages. Dev Cell 23, 1219-1229 (2012).

11. T. Nakamura, A. R. Gehrke, J. Lemberg, J. Szymaszek, N. H. Shubin, Digits and fin rays share common developmental histories. Nature 537, 225-228 (2016).

12. P. Haffter et al., Mutations affecting pigmentation and shape of the adult zebrafish. Dev Genes Evol 206, 260-276 (1996).

13. K. Henke et al., Genetic Screen for Postembryonic Development in the Zebrafish (Danio rerio): Dominant Mutations Affecting Adult Form. Genetics 207, 609-623 (2017).

14. H. Grandel, S. Schulte-Merker, The development of the paired fins in the zebrafish (Danio rerio). Mech Dev 79, 99-120 (1998).

15. M. E. Bowen, K. Henke, K. R. Siegfried, M. L. Warman, M. P. Harris, Efficient mapping and cloning of mutations in zebrafish by low-coverage whole-genome sequencing. Genetics 190, 1017-1024 (2012).

16. S. B. Snapper, F. S. Rosen, The Wiskott-Aldrich syndrome protein (WASP): roles in signaling and cytoskeletal organization. Annu Rev Immunol 17, 905-929 (1999).

17. X. Wu et al., Regulation of RNA-polymerase-II-dependent transcription by N-WASP and its nuclear-binding partners. Nat Cell Biol 8, 756-763 (2006).

18. C. Ferrai et al., Induction of HoxB transcription by retinoic acid requires actin polymerization. Mol Biol Cell 20, 3543-3551 (2009).

19. S. Suetsugu, T. Takenawa, Translocation of N-WASP by nuclear localization and export signals into the nucleus modulates expression of HSP90. J Biol Chem 278, 42515-42523 (2003).

20. F. J. van Eeden et al., Genetic analysis of fin formation in the zebrafish, Danio rerio. Development 123, 255-262 (1996).

21. X. R. Bustelo, Vav proteins, adaptors and cell signaling. Oncogene 20, 6372-6381 (2001). 
22. X. R. Bustelo, Vav family exchange factors: an integrated regulatory and functional view. Small GTPases 5, 9 (2014).

23. I. Hornstein, A. Alcover, S. Katzav, Vav proteins, masters of the world of cytoskeleton organization. Cell Signal 16, 1-11 (2004).

24. K. Fujikawa et al., Vav1/2/3-null mice define an essential role for Vav family proteins in lymphocyte development and activation but a differential requirement in MAPK signaling in T and B cells. $J$ Exp Med 198, 1595-1608 (2003).

25. K. Abe et al., Vav2 is an activator of Cdc42, Rac1, and RhoA. J Biol Chem 275, 1014110149 (2000).

26. M. F. Carlier, A. Ducruix, D. Pantaloni, Signalling to actin: the Cdc42-N-WASP-Arp2/3 connection. Chem Biol 6, R235-240 (1999).

27. T. Yano, G. Abe, H. Yokoyama, K. Kawakami, K. Tamura, Mechanism of pectoral fin outgrowth in zebrafish development. Development 139, 2916-2925 (2012).

28. P. Thorogood, in Developmental Patterning of the Vertebrate Limb, H. J. M. Hinchliffe J.R., Summerbell D., Ed. (Springer, Boston, 1991), vol. 205.

29. R. L. Lalonde, M. A. Akimenko, Effects of fin fold mesenchyme ablation on fin development in zebrafish. PLoS One 13, e0192500 (2018).

30. W. Masselink et al., A somitic contribution to the apical ectodermal ridge is essential for fin formation. Nature 535, 542-546 (2016).

31. J. Dewit, P. E. Witten, A. Huysseune, The mechanism of cartilage subdivision in the reorganization of the zebrafish pectoral fin endoskeleton. J Exp Zool B Mol Dev Evol 316, 584-597 (2011).

32. B. Hall, Bones and cartilage: Developmental and evolutionary skeletal biology. (Elsevier, Boston, ed. 2nd, 2015), pp. 892.

33. H. M. Kronenberg, Developmental regulation of the growth plate. Nature 423, 332-336 (2003).

34. R. W. Haines, The evolution of epiphyses and of endochondral bone. Biological Reviews of the Cambridge Philosophical Society 17, 267-292 (1942).

35. A. Askary et al., Ancient origin of lubricated joints in bony vertebrates. Elife 5, (2016).

36. D. K. Rhee et al., The secreted glycoprotein lubricin protects cartilage surfaces and inhibits synovial cell overgrowth. J Clin Invest 115, 622-631 (2005).

37. R. Diogo, J. Ziermann, J. Molnar, N. Siomava, V. Abdala, Muscles of Chordates: Development, Homologies, and Evolution. (CRC Press, 2018).

38. R. Winterbottom, Descriptive synonymy of the striated muscles of the Teleostei. Proc. Nat. Acad. Sciences 125, 225-317 (1973).

39. S. B. Snapper et al., N-WASP deficiency reveals distinct pathways for cell surface projections and microbial actin-based motility. Nat Cell Biol 3, 897-904 (2001).

40. M. Logan et al., Expression of Cre Recombinase in the developing mouse limb bud driven by a Prxl enhancer. Genesis 33, 77-80 (2002).

41. V. Cotta-de-Almeida et al., Wiskott Aldrich syndrome protein (WASP) and N-WASP are critical for T cell development. Proc Natl Acad Sci U S A 104, 15424-15429 (2007).

42. K. M. Small, S. S. Potter, Homeotic transformations and limb defects in Hox A11 mutant mice. Genes Dev 7, 2318-2328 (1993).

43. H. Haack, P. Gruss, The establishment of murine Hox-1 expression domains during patterning of the limb. Dev Biol 157, 410-422 (1993). 
44. C. E. Nelson et al., Analysis of Hox gene expression in the chick limb bud. Development 122, 1449-1466 (1996).

45. Y. Yokouchi, H. Sasaki, A. Kuroiwa, Homeobox gene expression correlated with the bifurcation process of limb cartilage development. Nature 353, 443-445 (1991).

46. A. P. Davis, D. P. Witte, H. M. Hsieh-Li, S. S. Potter, M. R. Capecchi, Absence of radius and ulna in mice lacking hoxa-11 and hoxd-11. Nature 375, 791-795 (1995).

47. C. Fromental-Ramain et al., Hoxa-13 and Hoxd-13 play a crucial role in the patterning of the limb autopod. Development 122, 2997-3011 (1996).

48. B. D. Metscher et al., Expression of Hoxa-11 and Hoxa-13 in the pectoral fin of a basal ray-finned fish, Polyodon spathula: implications for the origin of tetrapod limbs. Evol Dev 7, 186-195 (2005).

49. P. Sordino, F. van der Hoeven, D. Duboule, Hox gene expression in teleost fins and the origin of vertebrate digits. Nature 375, 678-681 (1995).

50. F. J. Tulenko et al., Fin-fold development in paddlefish and catshark and implications for the evolution of the autopod. Proc Biol Sci 284, (2017).

51. D. Duboule, Patterning in the vertebrate limb. Curr Opin Genet Dev 1, 211-216 (1991).

52. Y. Kherdjemil et al., Evolution of Hoxa11 regulation in vertebrates is linked to the pentadactyl state. Nature 539, 89-92 (2016).

53. J. M. Woltering, D. Noordermeer, M. Leleu, D. Duboule, Conservation and divergence of regulatory strategies at Hox Loci and the origin of tetrapod digits. PLoS Biol 12, e1001773 (2014).

54. C. Gegenbaur, Elements of Comparative Anatomy. (Macmllan \& Co, London, 1878), pp. 690.

55. F. J. Tulenko et al., HoxD expression in the fin-fold compartment of basal gnathostomes and implications for paired appendage evolution. Sci Rep 6, 22720 (2016).

56. H. Grandel, Approaches to a comparison of fin and limb structure and development. Theory in Biosciences 122, 288-301 (2003).

57. M. I. Coates, The origin of vertebrate limbs. Dev Suppl, 169-180 (1994).

58. C. Nusslein-Volhard, R. Dahm, Zebrafish: a practical approach. (Oxford University Press, Oxford 2002).

59. R. N. Kettleborough et al., A systematic genome-wide analysis of zebrafish proteincoding gene function. Nature 496, 494-497 (2013).

60. R_Core_Team, in $R$ Foundation for Statistical Computing. (Vienna, Austria, 2014).

61. W. R. Jackman, B. W. Draper, D. W. Stock, Fgf signaling is required for zebrafish tooth development. Dev Biol 274, 139-157 (2004).

62. A. Smith et al., Gene expression analysis on sections of zebrafish regenerating fins reveals limitations in the whole-mount in situ hybridization method. Dev Dyn 237, 417425 (2008). 


\section{Acknowledgements}

2 The authors would like to thank Drs. Joana Lopes and Matthew Warman for assistance in cell

3 culture and mouse husbandry, Dr. Neil Shubin for providing hox13 zebrafish mutants, and Dr.

4 Scott Snapper for sharing the Wasl conditional mouse. The authors would like to thank Drs.

5 Randall Dahn, Seth Donoughe, and James Hanken for their comments on early drafts of the

6 manuscript. This work was supported in part through support from Children's Orthopedic

7 Research Foundation, graduate research funds from the department of Organismic \&

8 Evolutionary Biology at Harvard University to MBH, and NSF DDIG 1600920.

9

\section{Author Contributions:}

$11 \mathrm{MBH}, \mathrm{KH}$, and MPH generated materials, designed the genetic and experimental approaches, 12 interpreted results, and wrote the paper. 
A

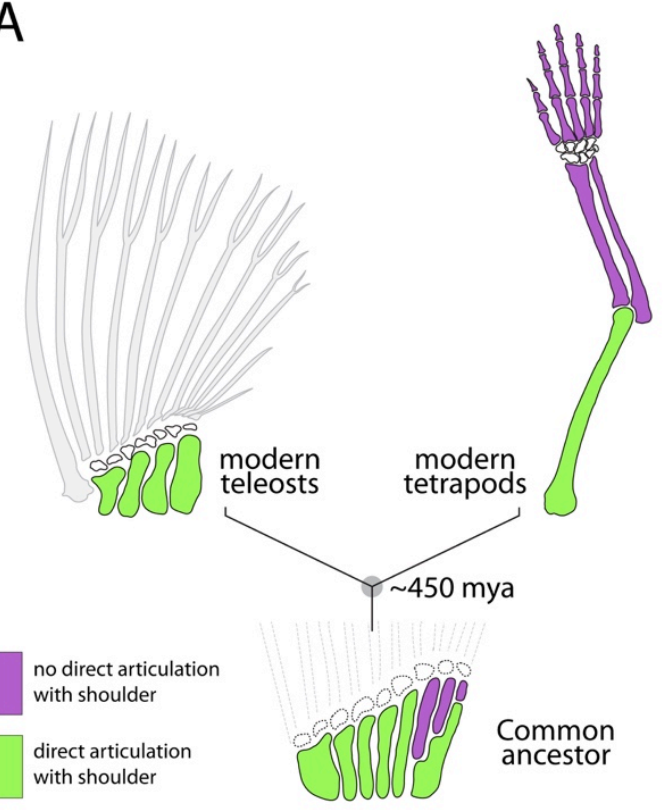

B wild type
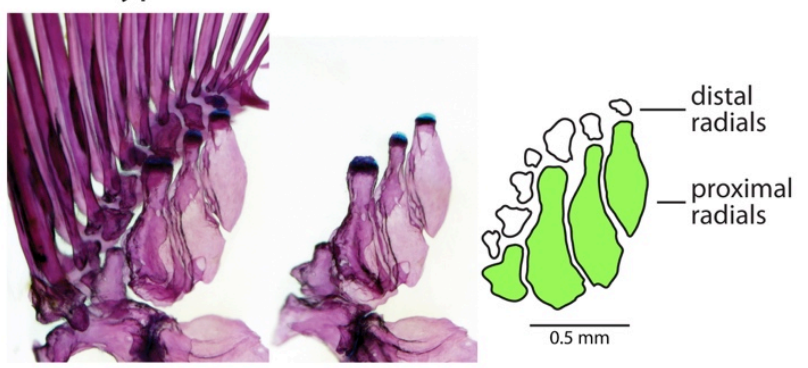

C rephaim +/-
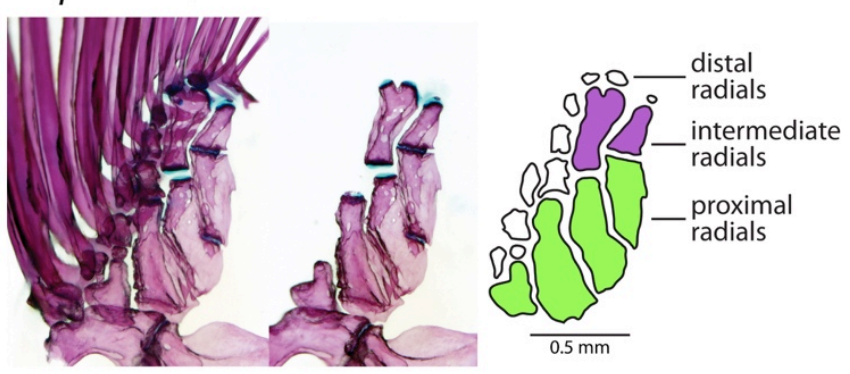
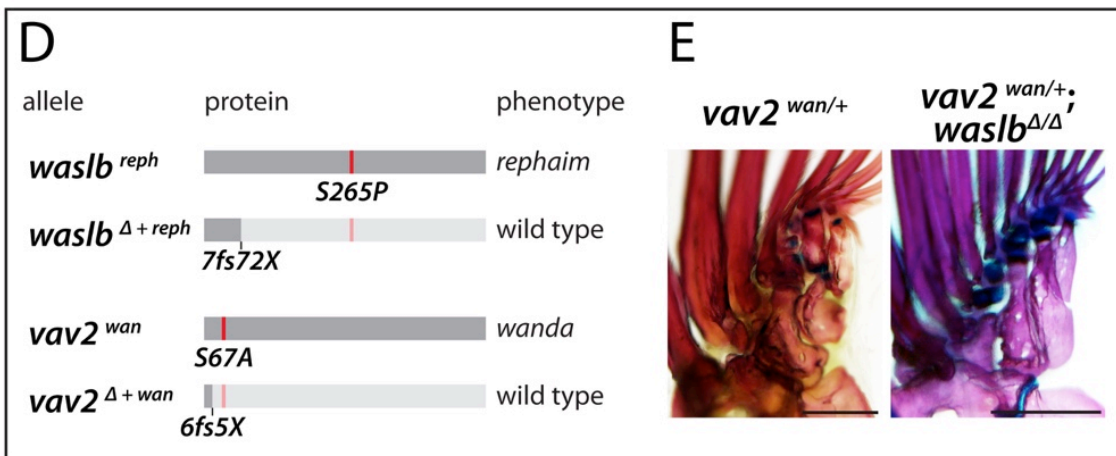

$\mathrm{F}$

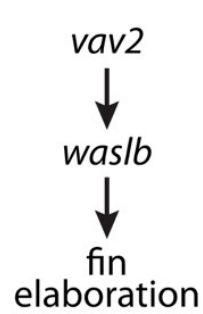

G

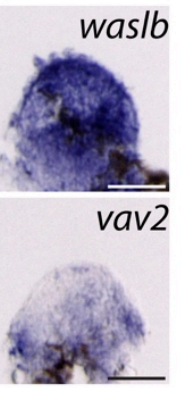

Figure 1. vav2 and waslb gain-of-function mutations reveal the capacity for limb-like development in fins. (A) From a common ancestor, teleosts and tetrapods evolved divergent appendage patterns. (B, C) Adult pectoral fin skeletons of wild-type (B) and heterozygous rephaim mutants $(\mathbf{C})$, shown intact (left), with fin rays and distal radials removed (center), and schematized (right). (D-G) Genetic characterization of reph and wan mutants. (D) Schematic of waslb and vav2 alleles found to cause reph and wan phenotypes, respectively. $\Delta+$ reph represents allele $m h 130$, and $\Delta+$ wan represents allele $m h 138$ (Figures S4, S5). (E) Epistasis analysis showing suppression of wan after the loss of waslb function. (F) Genetic pathway of 9 vav2, waslb, and fin elaboration. (G) Expression of waslb and vav2 transcripts in 48 hpf pectoral fin buds. Anterior to left, distal to top; scale bars (B, C, E) $500 \mu \mathrm{m},(\mathrm{G}) 50 \mu \mathrm{m}$. 

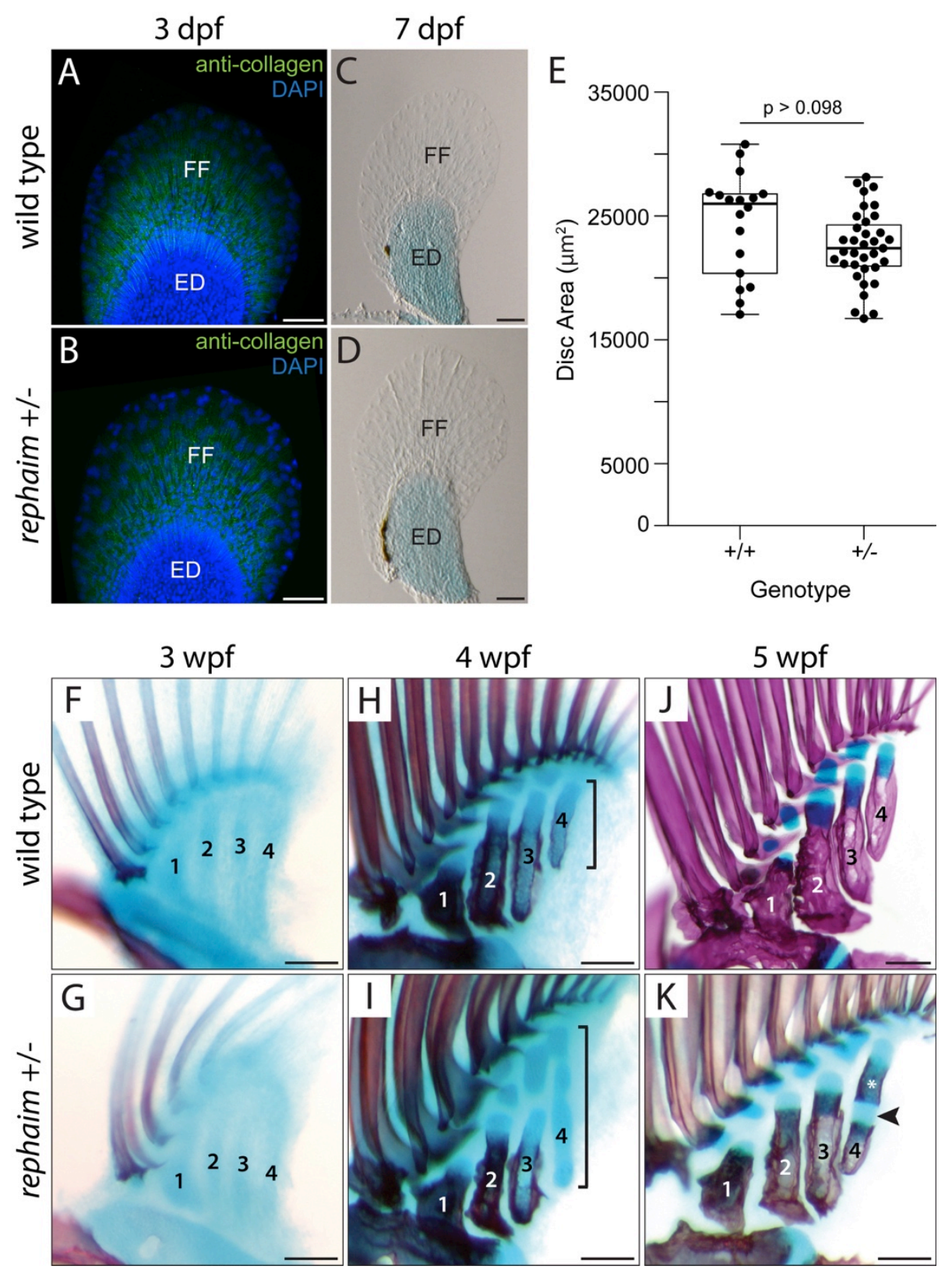

1 Figure 2. Novel skeletal elements arise by segmentation of a common cartilage precursor.

2 (A, B) Anti-collagen immunolabeling of actinotrichia fibers ( $\mathrm{n}=4$ for both genotypes). (C, D)

3 Alcian-stained pectoral fins. (E) Endoskeletal disc area at $7 \mathrm{dpf}$ is not significantly different

4 between wild-type and $\operatorname{reph}^{+/-}$fish (wild type $\mathrm{n}=18, \operatorname{reph}^{+/-} \mathrm{n}=35$, Welch's Two Sample $t$-test, $t=$

$5 \quad$-1.7145, p > 0.098). (F-K) Fin skeleton ontogeny in wild-type $(\mathbf{F}, \mathbf{H}, \mathbf{J})$ and $\operatorname{reph}^{+/}(\mathbf{G}, \mathbf{I}, \mathbf{K})$ fish

$6 \quad(n=5$ for both genotypes at each age). $(\mathbf{F}, \mathbf{G})$ Separation of the endoskeletal disc into four radial

7 precursors. $(\mathbf{H}, \mathbf{I})$ Proximal mineralization of the radials. Posterior radial cartilages are elongated

8 in reph $^{+/-}$mutants (brackets). ( $\left.\mathbf{J}\right)$ wild-type proximal radials exhibit continuous zones of

9 mineralization. (K) $\mathrm{reph}^{+/-}$mutants develop a secondary bone collar (asterisk) separated from the

10 proximal bone by cartilage (arrowhead). Anterior to left, distal to top; ED, endoskeletal disc; FF,

11 fin fold; 1-4, proximal radials; scale bars (A-D) $50 \mu \mathrm{m},(\mathrm{F}-\mathrm{K}) 125 \mu \mathrm{m}$. 


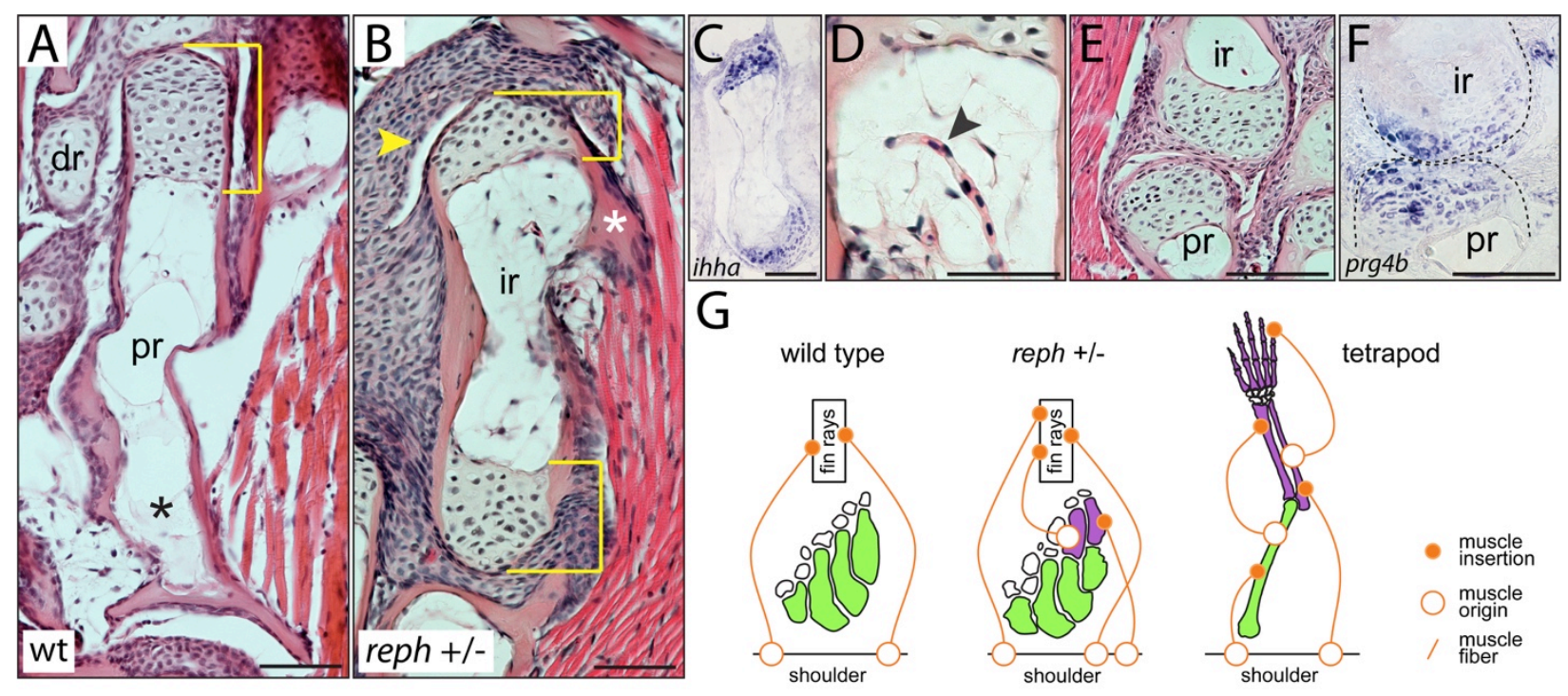

$1 \quad$ Figure 3. Intermediate radials are long bones morphologically integrated into the fin

2 skeleton. (A-D) Fin endoskeleton histology in adult wild-type and $r^{2 e p h}{ }^{+/}$adults. (A) Wild-type

3 proximal radial with a single distal epiphysis (bracket), and no proximal epiphysis (asterisk). (B)

4 Intermediate radial of $\mathrm{reph}^{+/-}$mutants have dual epiphyses (brackets), a muscle insertion point

5 (asterisk), and a synovial cavity (arrowhead). (C) in situ hybridization of chondrogenic marker

6 ihha in dual epiphysial compartments of an intermediate radial. (D) Blood vessel invading an

7 intermediate radial (arrowhead). (E) Histology of joint between intermediate and proximal

8 radials. (F) in situ hybridization of terminal joint marker prg $4 b$ expression in the proximal radial-

9 intermediate radial joint. (G) Diagram of muscle attachment in the tetrapod limb versus the

10 pectoral fins of wild-type and reph mutant zebrafish. Green, skeletal elements attaching to the

11 shoulder; purple, secondary branching elements; white, distal radials and carpals; dr, distal

12 radial; ir, intermediate radial; pr, proximal radial; anterior to left, distal to top; scale bars $50 \mu \mathrm{m}$. 


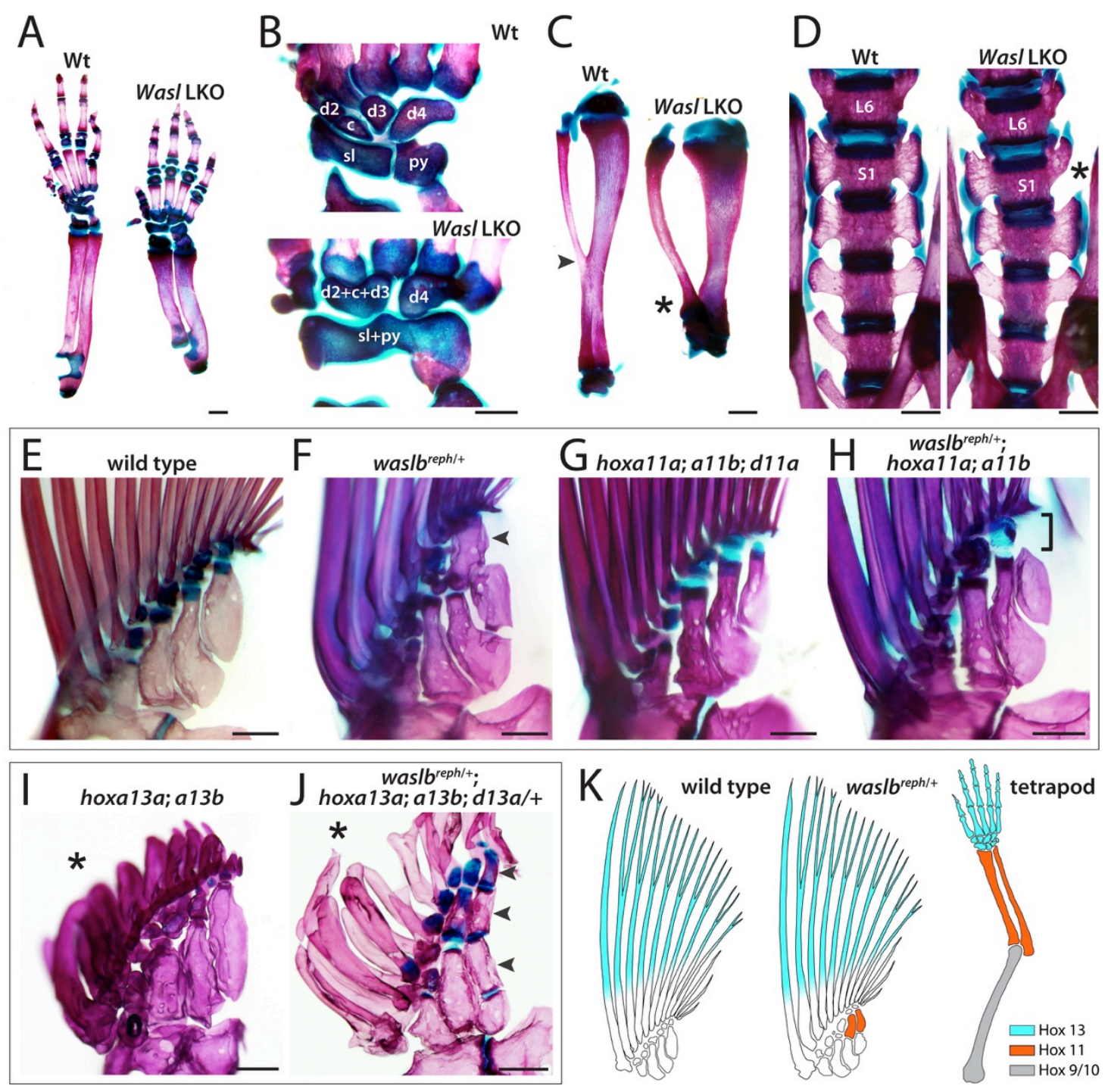

Figure 4. Wasl is required for normal limb patterning and interacts genetically with Hox genes to pattern fins. (A-D) Skeletal analysis of Prrx 1-Cre; Wasl ${ }^{\mathrm{L} 2 \mathrm{~L} / \mathrm{L} 2 \mathrm{~L}}$ (LKO) mice. (A) Limb phenotype of LKO and wild-type littermates. (B) Carpal fusions in the wrist and (C) reduced length and failed fusion of tibia and fibula in LKO mice. (D) Homeosis in sacral vertebrae in LKO mice. (E-J) Genetic interaction between waslb and hox genes in zebrafish fin patterning. (E) wild-type adult. (F) waslb ${ }^{\text {reph/+ }}$ mutants with wild-type hox 11 paralogs form intermediate radials (arrowhead; $\mathrm{n}=9,89 \%) .(\mathbf{G})$ hoxal $1 a^{-/-}$; hoxal $1 b^{-/-}$; hoxd1 $1 a^{-/-}$mutants have normal pectoral fins. (H) waslb ${ }^{\text {reph/+ }}$; hoxal $1 a^{-/-}$; hoxal $1 b^{-/-}$mutants fail to form intermediate radials (bracket; $\mathrm{n}=8,75 \%$ ). (I) hoxa $13 a^{-/-}$; hoxa13 $b^{-/-}$mutants have defective and reduced fin rays (asterisk) but maintain a wild-type proximal radial pattern. (J) waslb $^{\text {reph/+ }}$; hoxal $3 a^{-/-}$; hoxal3b $b^{-/-}$; hoxd $13 a^{+/-}$mutants have enhanced PD elaboration with multiple intermediate radials (arrowheads). (K) Model of region-specific requirements of Hox paralogs in PD patterning of fin and limb bones as revealed by Hox null mutations in zebrafish and mouse. Anterior to left, distal to top; c, central carpal; d2-4, distal carpals; py, pyramidal; L6, lumbar vertebrae 6; S1, sacral vertebrae 1; sl, scapholunate; scale bars (A, C, E) 1 mm, (B) $500 \mu \mathrm{m}$, (E-J) $250 \mu \mathrm{m}$. 


\section{Supplemental Data}

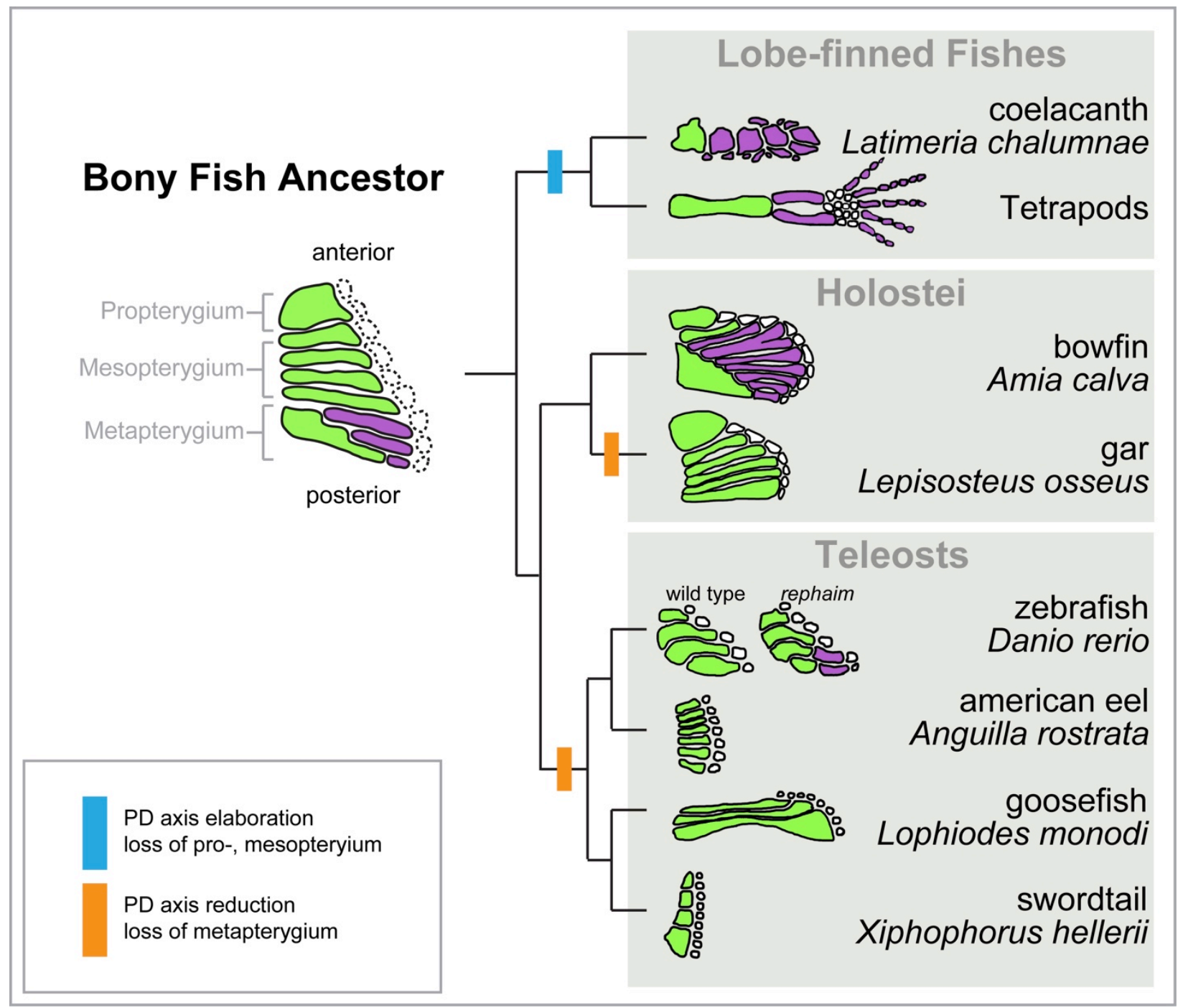

1 Supplemental Figure 1. Phylogenetic model of pectoral appendage articulation in bony

2 fishes. The last common ancestor of the bony fishes, the clade that encompasses the teleost and 3 tetrapod lineages, had a polybasal condition in which the most proximal endochondral elements

4 of the fin articulated with the shoulder girdle. The pectoral fin consisted of the anterior

5 propterygium, the middle mesopterygium, and the posterior metapterygium. In the lineage

6 leading to tetrapods, the propterygium and mesopterygium are postulated to have been lost along

7 with the dermal fin rays, while new long bones that articulate end on end were added along the

8 PD axis of the metapterygium, ultimately forming the limb. Conversely, in the teleost lineage, it

9 is proposed that the metapterygium was lost while the propterygium and mesopterygium were

10 retained, and no teleost exhibits end-on-end articulation of long bones in the pectoral fin. While

11 rare, variation in the teleost pectoral fin endoskeleton is observed in proximal radial length as

12 well as the number of radials along the AP axis of the fin in different clades. However, no teleost

13 species showing PD long bone articulation comparable to that seen in tetrapods have been

14 described. 


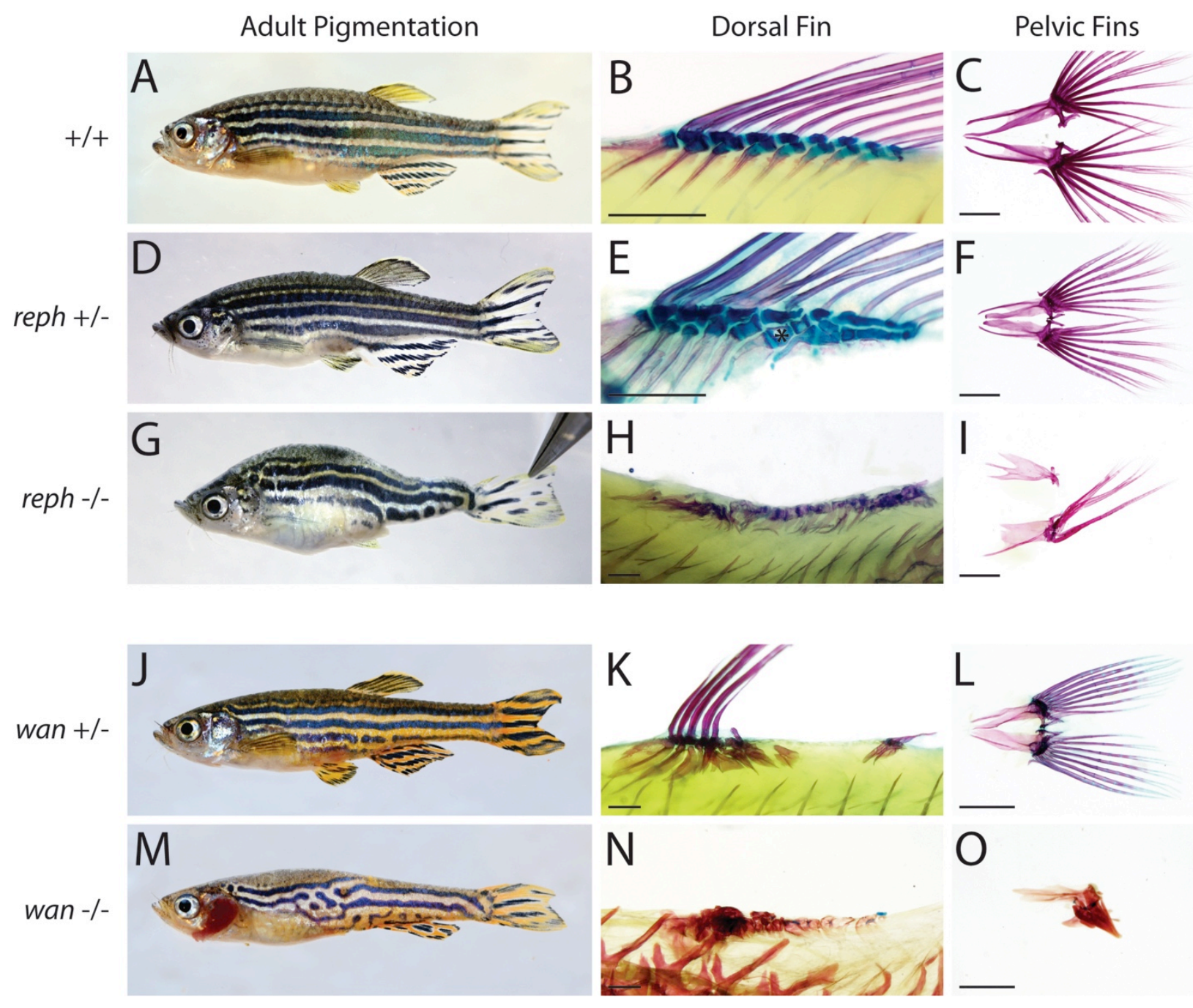

Supplemental Figure 2. Additional adult phenotypes of reph and wan mutants. Adult pigmentation and skeletal patterning phenotypes of dorsal and pelvic fins in reph and wan mutants. (A-C) wild-type adult. (D-F) reph heterozygotes show increased number of radials in the dorsal fin (asterisk), however pelvic fins (F) are unaffected. (G-I) reph homozygous mutants show more severe effect on pigmentation $(\mathbf{G})$, and defects in lepidotrichia growth and maintenance $(\mathbf{H}, \mathbf{I})$. Dorsal fin endoskeleton in homozygous fish is uninterpretable. (J-L) wan heterozygotes show alteration in pigmentation $(\mathbf{J})$, and medial fin growth and patterning $(\mathbf{K})$.

8 Pelvic fins are unaffected (L). (M-O) Similar to reph, wan homozygous fish show a more severe 9 effect on pigmentation (M), and highly dysmorphic fin rays and endoskeletal elements in the 10 dorsal and pelvic fins (N, O). Scale bars (B, E, H, K, N) $500 \mu \mathrm{m},(\mathrm{C}, \mathrm{F}, \mathrm{I}, \mathrm{L}, \mathrm{O}) 1 \mathrm{~mm}$. 

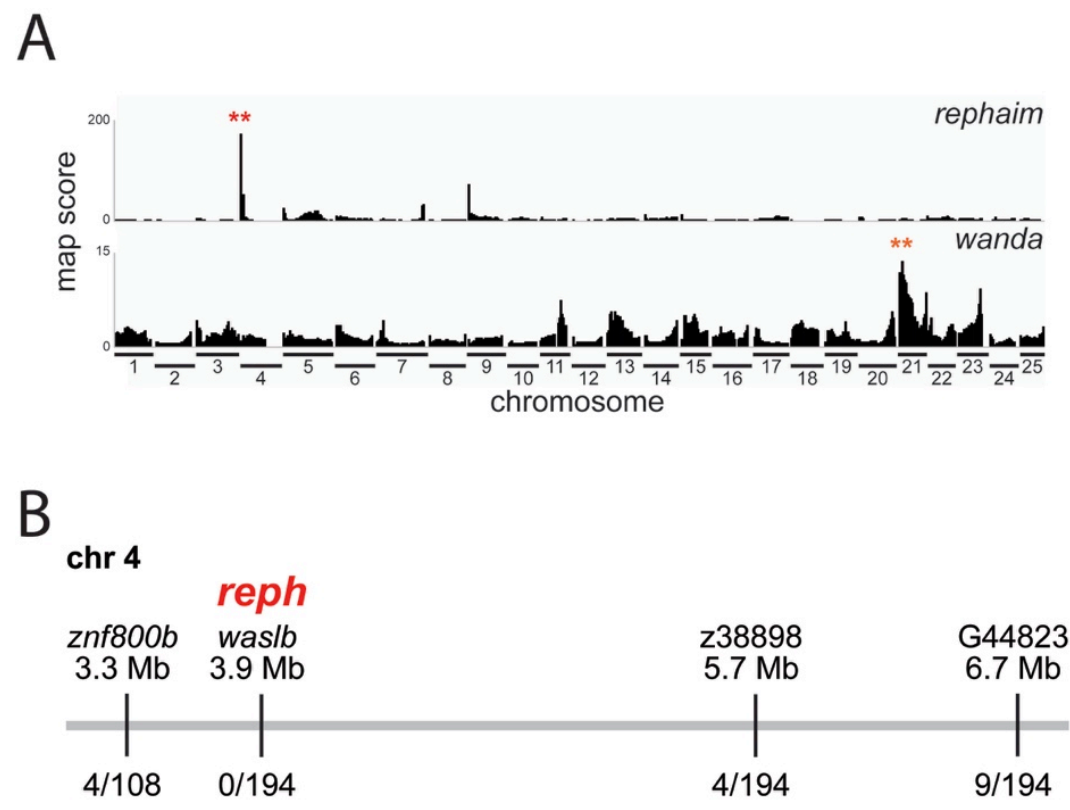

chr 21

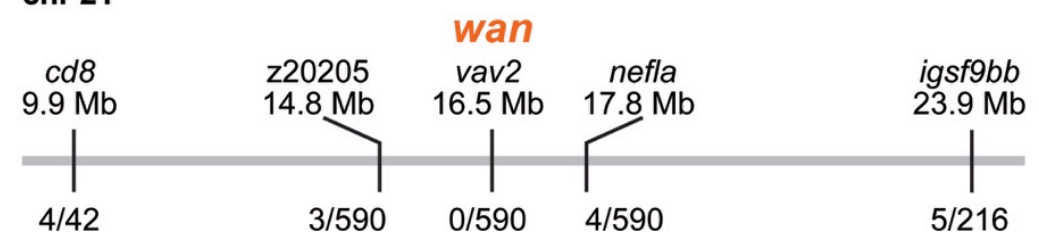

1 Supplemental Figure 3. Mapping the causative mutations underlying the rephaim and

2 wanda phenotypes. (A) Mapping score from homozygosity-by-descent analysis for reph and

3 wan mutants based on whole-exome sequencing of homozygous populations. Asterisks highlight

4 regions of putative linkage. (B) Fine mapping of the chromosome with the highest mapping

5 score by recombination analysis in individual homozygous mutants. Shown are informative

6 markers used, their position, and the number of recombinants identified per meiosis scored.

7 Within the smaller linked interval, sequence data from the mutant pool was used to identify

8 nonsynonymous SNPs as candidate mutations responsible for the altered skeletal phenotype of

9 reph and wan. 

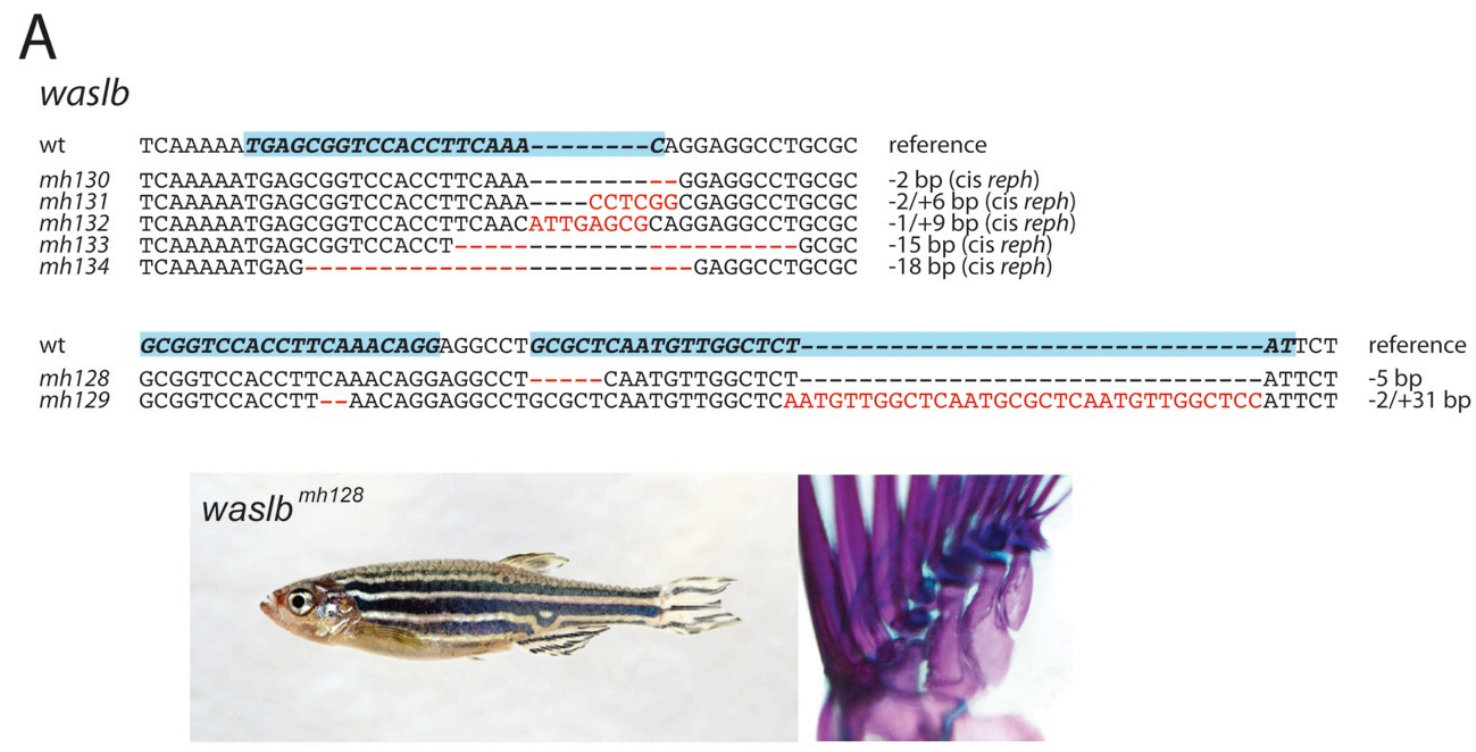

B

vav2

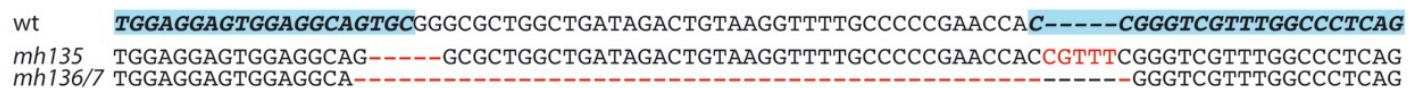
mh138 TGGAGGAGTGGAGG----GCGGGCGCTGGCTGATAGACTGTAAGGTTTTGCCCCCGAACCAC-----CGGGTCGTTTGGCCTCAG reference
$-5 / 5$ bp
$-47 \mathrm{bb}$ (wt \& wan)
$-4 \mathrm{bp}$ (wan)
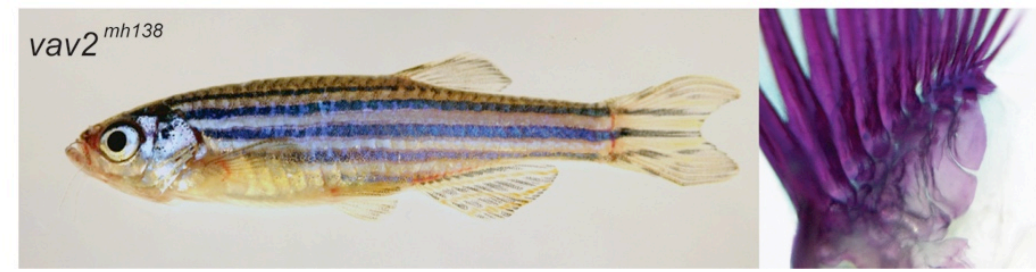

1 Supplemental Figure 4. CRISPR-generated loss-of-function mutants of waslb and vav2.

2 Mutant lines of (A) waslb and (B) vav2 generated by CRISPR-Cas9 targeted gene editing.

3 Shown is the targeted wild-type sequence as well as the sequence for the generated mutant

4 alleles (blue indicates guide target sequence). Pictures show representative homozygous mutants.

5 All mutants are homozygous viable and fertile. Whole mount skeletal stained adult pectoral fins

6 from homozygous mutants show no obvious defects in fin patterning. 
A

Waslb

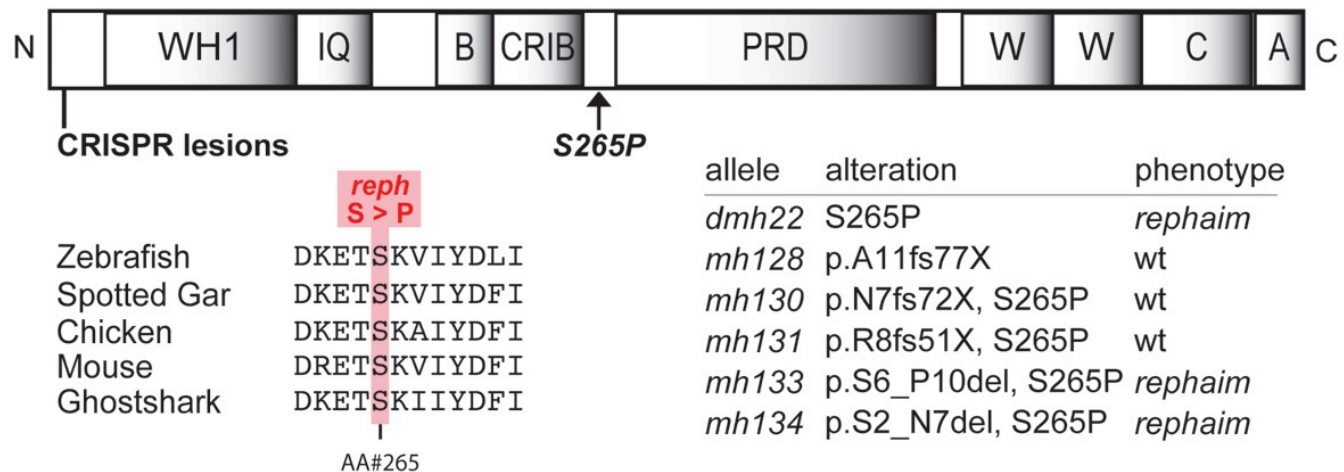

\section{B}

Vav2

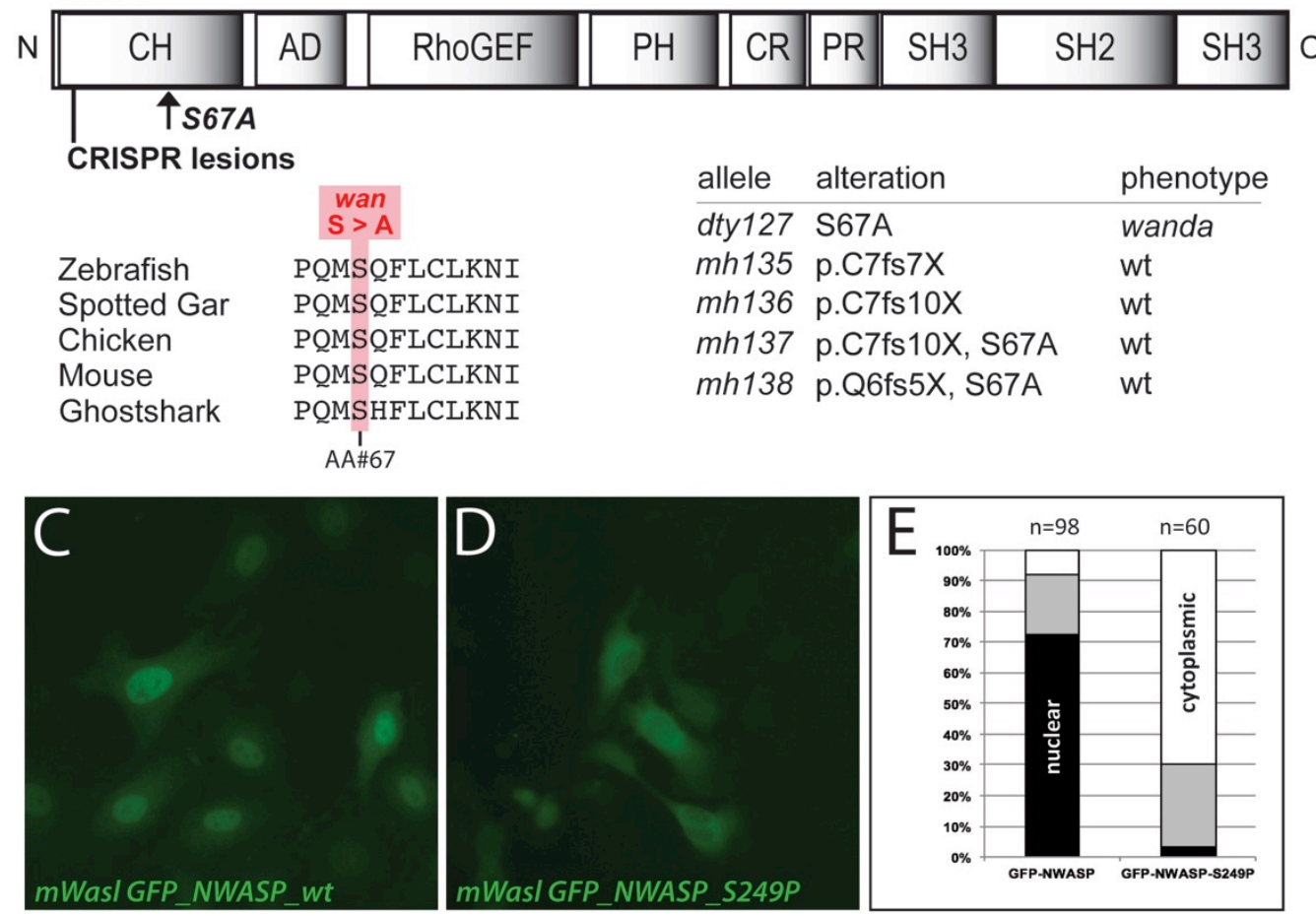

$1 \quad$ Supplemental Figure 5. Gain-of-function mutations in waslb and vav2 cause the reph and wan phenotypes, respectively. (A) Waslb and (B) Vav2 protein schematics showing positions 3 of missense mutations and CRISPR-generated lesions. Multiple sequence alignment reveals 4 conservation of the mutated residues across vertebrates. Mutant alleles are listed with their 5 consequence on amino acid sequence and their phenotypic effect. (C-D) HeLa cells transfected 6 with constructs encoding N-terminal GFP fusions to mouse wild-type N-Wasp (C) and an N7 Wasp version containing the orthologous reph mutation (S249P) (D). (E) Qualitative scoring of 8 transfected cells; white, predominantly cytoplasmic localization; black, predominantly nuclear 9 localization; grey, no bias observed in localization. 


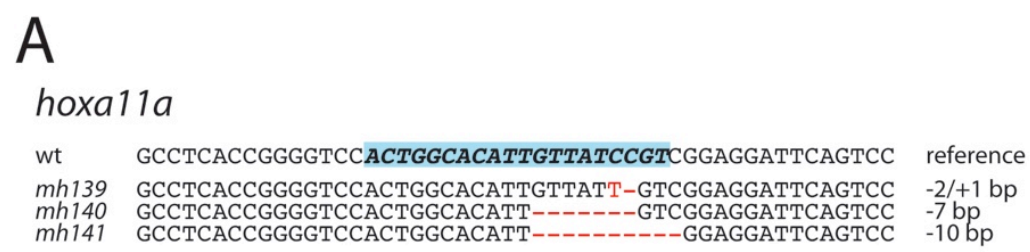

B

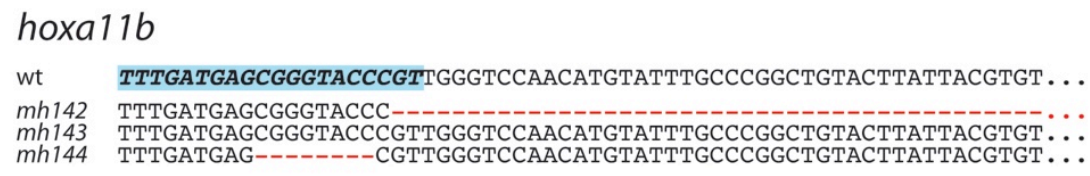

C

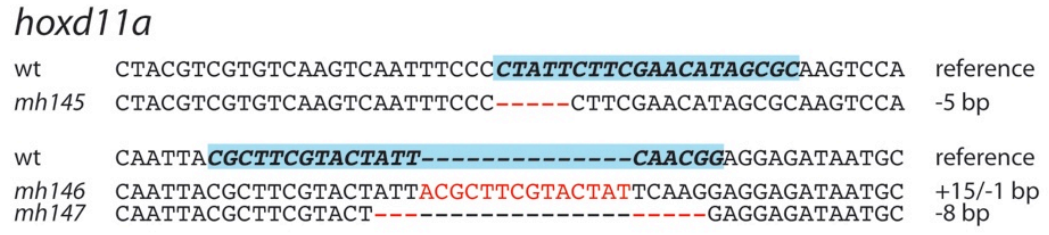

D
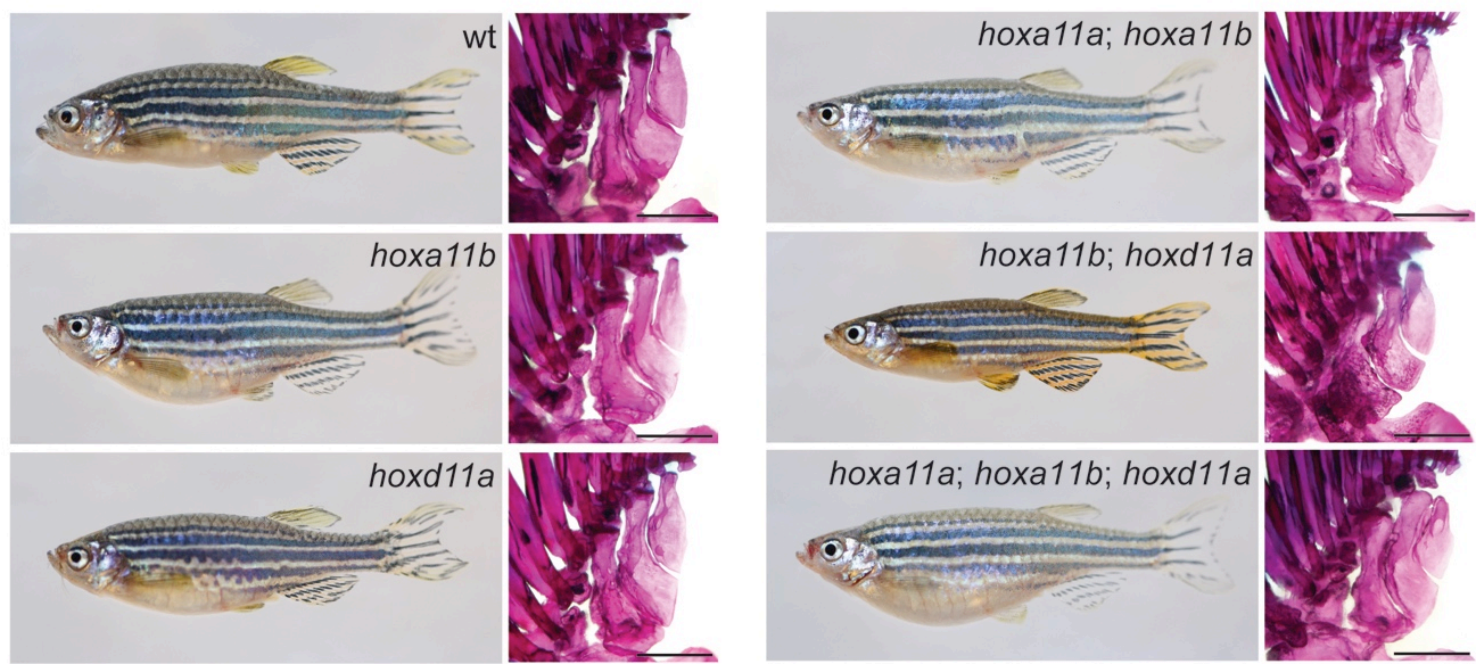

1 Supplemental Figure 6. CRISPR-generated hox11 loss-of-function mutants.

2 Mutant lines of hox11 paralogs (A) hoxalla, (B) hoxal 1b, and (C) hoxdl la generated by

3 CRISPR-Cas9 targeted gene editing. Shown is the targeted wild-type sequence as well as the 4 sequence for the generated mutant alleles (blue indicates guide target sequence).

5 (D) Representative singular and compound mutants. All mutant combinations are viable and 6 fertile. Scale bars $500 \mu \mathrm{m}$. 


\section{Supplemental Table 1. Skeletal phenotypes in Wasl LKO mice}

2

\begin{tabular}{lcc}
\hline & $\begin{array}{c}\text { wild type } \\
(\mathrm{n}=5)\end{array}$ & $\begin{array}{c}\text { Wasl LKO } \\
(\mathrm{n}=5)\end{array}$ \\
\hline Vertebral pattern & 5 & 2 \\
6 Lumbar / 4 Sacral (wild type) & 0 & 2 \\
6 Lumbar / 1 Mixed Identity / 3 Sacral & 0 & 1 \\
7 Lumbar / 3 Sacral & & \\
Wrist pattern & & 0 \\
normal & 5 & 5 \\
d2 + c + d4 fusion & 0 & 5 \\
sl + py fusion & 0 & $0 / 5$ \\
pi + T fusion & 0 & \\
& & $0 / 5$ \\
Tibia and Fibula: normal/unfused & $5 / 0$ & \\
Limb long bones: normal/shortened & & $5 / 0$ \\
\hline
\end{tabular}

3

4 Counts reported for limb and axial phenotypes observed in Wasl LKO mice and wild-type

5 littermates cleared and stained at P30. c, central carpal; d2, distal carpal 2; d4, distal carpal 4; pi,

6 pisiform; py, pyramidal; sl, scapholunate; T, triangular. 